\title{
VIRASORO SYMMETRY OF THE CONSTRAINED MULTI-COMPONENT KP HIERARCHY AND ITS INTEGRABLE DISCRETIZATION
}

\author{
CHUANZHONG LI $\dagger$, JINGSONG HE $\ddagger$ \\ Department of Mathematics, Ningbo University, Ningbo, 315211, China \\ †email:lichuanzhong@nbu.edu.cn \\ łemail:hejingsong@nbu.edu.cn
}

\begin{abstract}
In this paper, we construct the Virasoro type additional symmetries of a kind of constrained multi-component KP hierarchy and give the Virasoro flow equation on eigenfunctions and adjoint eigenfunctions. It can also be seen that the algebraic structure of the Virasoro symmetry is kept after discretization from the constrained multi-component KP hierarchy to the discrete constrained multi-component KP hierarchy.
\end{abstract}

Mathematics Subject Classifications(2000): 37K05, 37K10, 37K40.

Keywords: constrained multi-component KP hierarchy, discrete constrained multi-component KP hierarchy, Virasoro symmetry

\section{Contents}

1. Introduction

2. The constrained multi-component KP hierarchy

3. Virasoro symmetry of the constrained multi-component KP hierarchy

4. The discrete constrained multi-component KP hierarchy

5. Virasoro symmetry of the discrete constrained multi-component KP hierarchy 14

6. Conclusions and Discussions

References

\section{INTRODUCTION}

The KP hierarchy is one of the most important nonlinear integrable system in mathematics and physics. The additional symmetry of the KP hierarchy was constructed by L. A. Dickey in [1] in which the additional symmetry constitutes a famous algebra called Virasoro algebra. The KP system becomes one of the most fundamental integrable models in mathematical physics as Toda system. As one of the most important sub-hierarchies of the KP hierarchy by considering different reduction conditions on the Lax operator, the constrained KP hierarchy contains a large number of interesting soliton equations under the so-called symmetry constraint [2, 3, 4] 
which means that the negative part of the Lax operator of the constrained KP hierarchy is a generator of the additional symmetries of the KP hierarchy. However, the additional symmetry flows of the KP hierarchy are not consistent with the special form of the Lax operator for the constrained KP hierarchy. Therefore it is highly non-trivial to find a suitable additional symmetry flows on eigenfunctions and adjoint eigenfunctions for this sub-hierarchy [5] to make the constraint compatible with its additional symmetry. In addition to the above-mentioned integrable systems, other KP type and Toda type systems also have interesting algebraic structure in additional symmetries [6, 7, 8, 9].

The multi-component KP hierarchy is important matrix-formed generalization of the original KP hierarchy and its additional symmetry was well-studied in [10]. In [11, the representation related to the multi-component $\mathrm{KP}$ hierarchy was proposed. In a certain sense, the multicomponent KP hierarchy served as a universal integrable hierarchy because many well known integrable hierarchies come from certain reductions of it, for example, like the Davey-Stewartson system, the two-dimensional Toda lattice, the $n$-wave system and the Darboux-Egoroff system. The Hirota bilinear equations of the multi-component KP hierarchy play an interesting role in the theory of theta functions of Riemann surfaces. To be more specific, it shows that the theta function of a Riemann surfaces gives a tau function for the $n$-component KP hierarchy in [12. It also show that such a theta function satisfies the Fay trisecant formula. There is a new interesting phenomenon in the $n$-component case which does not occur in the single-component case: the tau function and the wave function are a collection of functions parameterized by the elements of the root lattice of type A. Later with reduction, the special but quite natural constrained multi-component KP hierarchy was studied by Y. Zhang in [13]. But for the additional symmetry of this reduced hierarchy it is still in blank and interesting. That becomes one motivation of us to do this work. Recently the research on random matrices and nonintersecting Brownian motions and the study of moment matrices with regard to several weights showed that the determinants of such moment matrices these determinants are tau-functions of the multi-component KP-hierarchy [14]. Basing on above mentioned importance of the multicomponent KP-hierarchy, it is quite necessary to go on further study on this hierarchy and its reduced hierarchy.

Besides above continuous dynamical systems, discrete integrable systems becomes more and more important. For multicomponent discrete integrable systems, the multicomponent Toda hierarchy recently attracts a lot of valuable research such as [15, 16, 17]. The Hamiltonian structures and tau function for the discrete KP hierarchy was introduced in ([18]-[20]). Later the additional symmtries and ghost symmetry for the discrete KP hierarchy was considered in [8, 21] respectively. In our recent work [9] the additional symmetries of one-component constrained discrete KP hierarchy was constructed in which a suitable additional symmetry flows on eigenfunctions and adjoint eigenfunctions for this hierarchy was given. In this paper, we also consider the discrete constrained multi-component KP hierarchy and identify its algebraic structure hidden behind this discrete hierarchy.

Our main purpose of this article is to identify the Virasoro symmetry of the constrained multicomponent KP hierarchy hierarchy and give the complete Virasoro flows on eigenfunctions and adjoint eigenfunctions in the Lax operator of the hierarchy which form the positive half of 
Virasoro algebra using the technique in [5, 9]. Also we give the Virasoro symmetry of the discrete constrained multi-component KP hierarchy. That shows that the algebraic structure of the Virasoro symmetry is kept after discretization from the constrained multi-component KP hierarchy to the discrete constrained multi-component KP hierarchy.

This paper is organized as follows. We give a brief description of the constrained $N$ component KP hierarchy in Section 2. The main results are stated and proved in Section 3, which concerns the Virasoro symmetry of the multi-component constrained KP hierarchy. Section 4 and Section 5 will concerns the Virasoro symmetry of the discrete multi-component constrained KP hierarchy. Section 6 is devoted to conclusions and discussions.

\section{The COnstrained MUlti-COMPOnEnt KP HIERARChY}

For an $N$-component KP hierarchy, there are $N$ infinite families of time variables $t_{\alpha n}, \alpha=$ $1, \ldots, N, n=1,2, \ldots$ The coefficients $u_{1}, u_{2}, \ldots$ of the Lax operator

$$
L=E \partial+u_{1} \partial^{-1}+u_{2} \partial^{-2}+\ldots
$$

are $N \times N$ matrices and $\partial$ is the derivative about spatial variable $x$. There are another $N$ pseudo-differential operators $R_{1}, \ldots, R_{N}$ in the matrix form

$$
R_{\alpha}=E_{\alpha}+u_{\alpha 1} \partial^{-1}+u_{\alpha 2} \partial^{-2}+\ldots
$$

where $E_{\alpha}$ is the $N \times N$ matrix with 1 on the $(\alpha, \alpha)$-component and zero elsewhere, and $u_{\alpha 1}, u_{\alpha 2}, \ldots$ are also $N \times N$ matrices. The operators $L, R_{1}, \ldots, R_{N}$ satisfy the following conditions:

$$
L R_{\alpha}=R_{\alpha} L, \quad R_{\alpha} R_{\beta}=\delta_{\alpha \beta} R_{\alpha}, \quad \sum_{\alpha=1}^{N} R_{\alpha}=E .
$$

The Lax equations of the $N$-component KP hierarchy are:

$$
\frac{\partial L}{\partial t_{\alpha, n}}=\left[B_{\alpha, n}, L\right], \quad \frac{\partial R_{\beta}}{\partial t_{\alpha, n}}=\left[B_{\alpha, n}, R_{\beta}\right], \quad B_{\alpha, n}:=\left(L^{n} R_{\alpha}\right)_{+} .
$$

The "+" means the projection onto the terms with the nonnegative powers about $\partial$. One can check that the operator $\partial$ now is equal to $\partial_{t_{11}}+\ldots+\partial_{t_{N 1}}$. In fact the Lax operator $L$ and $R_{\alpha}$ can have the following dressing structures

$$
L=S \partial S^{-1}, \quad R_{\alpha}=S E_{\alpha} S^{-1}
$$

where

$$
S=E+\sum_{i=1}^{\infty} S_{i} \partial^{-i}
$$

and $S_{1}$ will be used to define eigenfunction $P^{(r)}$ and adjoint eigenfunction $Q^{(r)}$ later in a constrained hierarchy. 
As [13], the following reduction condition is imposed onto the $N$-component KP hierarchy:

$$
\sum_{l=1}^{r} d_{l}^{s}\left(L R_{l}\right)^{s}+\sum_{l=r+1}^{N} L R_{l}=\sum_{l=1}^{r} d_{l}^{s}\left(L R_{l}\right)_{+}^{s}+\sum_{l=r+1}^{N}\left(L R_{l}\right)_{+}, \quad 1 \leq r \leq N .
$$

Let us denote following operators as 13

$$
\begin{gathered}
\hat{\partial}:=\partial_{1}+\partial_{1}+\cdots+\partial_{r}, \quad \partial_{i}=\frac{\partial}{\partial t_{i 1}} \\
\hat{L}^{(i)}=S^{(r)}(\hat{\partial}) E_{i}^{(r)} \hat{\partial}\left(S^{(r)}(\hat{\partial})\right)^{-1}, \quad \hat{L}=\sum_{i=1}^{r} d_{i} \hat{L}^{(i)},
\end{gathered}
$$

and

$$
\begin{aligned}
B_{k}^{(l, r)} & =\left(S^{(r)}(\hat{\partial}) E_{l}^{(r)} \hat{\partial}^{k}\left(S^{(r)}(\hat{\partial})\right)^{-1}\right)_{+}:=\left(C_{k}^{(l, r)}\right)_{+}, \quad 1 \leq l \leq r, \\
C_{k}^{(l, r)} & =S^{(r)}(\hat{\partial}) E_{l}^{(r)} \hat{\partial}^{k}\left(S^{(r)}(\hat{\partial})\right)^{-1}, \quad 1 \leq l \leq r, \\
P^{(r)} & =\left.\left(S_{1}\right)_{i j}\right|_{1 \leq i \leq r, r+1 \leq j \leq N}, \quad Q^{(r)}=\left.\left(S_{1}\right)_{i j}\right|_{1 \leq j \leq r, r+1 \leq i \leq N},
\end{aligned}
$$

where $S^{(r)}\left(E_{i}^{(r)}\right)$ is the $r \times r$ principal sub-matrix of $S\left(E_{i}\right)$ and the projection " + " here is about the operator $\hat{\partial}$. This further lead to

$$
\mathcal{L}=\hat{L}^{s}=\sum_{l=1}^{r} d_{l}^{s} B_{s}^{(l, r)}+P^{(r)} \hat{\partial}^{-1} Q^{(r)} .
$$

Under the constraint eq.(2.4), the following evolutionary equations of the constrained $N$ component KP hierarchy can be derived [13].

Proposition 2.1. For the constrained $N$-component KP hierarchy, the following hierarchies of equations can be derived [13]

$$
\begin{gathered}
\frac{\partial P^{(r)}}{\partial t_{n l}}=B_{n}^{(l, r)} P^{(r)}, \quad \frac{\partial Q^{(r)}}{\partial t_{n l}}=-\left(B_{n}^{(l, r)}\right)^{*} Q^{(r)}, \\
\frac{\partial \hat{L}^{s}}{\partial t_{n l}}=\left[B_{n}^{(l, r)}, \hat{L}^{s}\right],
\end{gathered}
$$

where for $B_{n}^{(l, r)}=\sum_{i=0}^{n} B_{i} \hat{\partial}^{i}$,

$$
\left(B_{n}^{(l, r)}\right)^{*} Q^{(r)}:=\sum_{i=0}^{n}(-1)^{i} \hat{\partial}^{i}\left(Q^{(r)} B_{i}\right)
$$

When $r=1, N=2$, one can obtain the $s$-constrained $\mathrm{KP}$ hierarchy [3, 4]. When $r=$ $1, N \geq 2$, one can obtain the vector $s$-constrained KP hierarchy[23]. For general $r, N$, the generalized constrained KP hierarchy can be obtained which can also be seen in the setting of the generalized Drinfeld-Sokolov construction [24, 25]. 


\section{VirAsoro SYMmETRY OF THE CONSTRAINED MULTI-COMPONENT KP HIERARCHY}

In this section, we shall construct the additional symmetry and discuss the algebraic structure of the additional symmetry flows of the $N$-component constrained KP hierarchy.

To this end, firstly we define $\hat{\Gamma}^{(r)}$ and the Orlov-Shulman's operator $\mathcal{M}$ like in [22]

$$
\hat{\Gamma}^{(r)}=\sum_{\beta=1}^{r} t_{1 \beta} \frac{E_{\beta}}{s d_{\beta}^{s}} \hat{\partial}^{1-s}+\sum_{\beta=1}^{r} \sum_{n=2}^{\infty} \frac{n}{s} d_{\beta}^{-s} E_{\beta} \hat{\partial}^{n-s} t_{n \beta}, \quad \mathcal{M}=S^{(r)} \hat{\Gamma}^{(r)}\left(S^{(r)}\right)^{-1} .
$$

It is easy to find the following formula

$$
\left[\partial_{t_{n \beta}}-\hat{\partial}^{n} E_{\beta}, \hat{\Gamma}^{(r)}\right]=0 .
$$

There are the following commutation relations

$$
\left[\sum_{\beta=1}^{r} d_{\beta}^{s} \hat{\partial}^{s} E_{\beta}, \hat{\Gamma}^{(r)}\right]=E,
$$

which can be verified by a straightforward calculation. By using the Sato equation, the isospectral flow of the $\mathcal{M}$ operator is given by

$$
\partial_{t_{n \beta}} \mathcal{M}=\left[B_{n}^{(\beta, r)}, \mathcal{M}\right] .
$$

More generally,

$$
\partial_{t_{n \beta}}\left(\mathcal{M}^{m} \mathcal{L}^{l}\right)=\left[B_{n}^{(\beta, r)}, \mathcal{M}^{m} \mathcal{L}^{l}\right] .
$$

The Lax operator $\mathcal{L}$ and the Orlov-Shulman's $\mathcal{M}$ operator satisfy the following canonical relation

$$
[\mathcal{L}, \mathcal{M}]=E \text {. }
$$

Then the additional flows for the time variable $t_{m, n, \beta}$ will be defined as follows

$$
\frac{\partial S}{\partial t_{m, n, \beta}}=-\left(\mathcal{M}^{m} C_{n}^{(\beta, r)}\right)_{-} S, \quad m, n \in \mathbb{N}, 1 \leq \beta \leq r .
$$

or equivalently

$$
\frac{\partial \mathcal{L}}{\partial t_{m, n, \beta}}=-\left[\left(\mathcal{M}^{m} C_{n}^{(\beta, r)}\right)_{-}, \mathcal{L}\right], \quad \frac{\partial \mathcal{M}}{\partial t_{m, n, \beta}}=-\left[\left(\mathcal{M}^{m} C_{n}^{(\beta, r)}\right)_{-}, \mathcal{M}\right] .
$$

Later we can prove the additional flows $\frac{\partial}{\partial t_{m, n, \beta}}$ commute with the flow $\frac{\partial}{\partial t_{k, \gamma}}$, i.e. $\left[\frac{\partial}{\partial t_{m, n, \beta}}, \frac{\partial}{\partial t_{k, \gamma}}\right]=$ 0 , but do not commute with each other. They form a kind of $W_{\infty}$ infinite dimensional additional Lie algebra symmetries which contain Virasoro algebra as a special subalgebra. To this purpose, we need several lemmas and propositions as preparation firstly.

For above local differential operators $B_{n}^{(\beta, r)}$, we have the following lemma.

Lemma 3.1. $\left[B_{n}^{(\beta, r)}, P^{(r)} \hat{\partial}^{-1} Q^{(r)}\right]_{-}=B_{n}^{(\beta, r)}\left(P^{(r)}\right) \hat{\partial}^{-1} Q^{(r)}-P^{(r)} \hat{\partial}^{-1} B_{n}^{(\beta, r) *}\left(Q^{(r)}\right)$. 
Proof. Firstly we consider a fundamental monomial: $\hat{\partial}^{n}(n \geq 1)$. Then

$$
\left[\hat{\partial}^{n}, P^{(r)} \hat{\partial}^{-1} Q^{(r)}\right]_{-}=\left(\hat{\partial}^{n}\left(P^{(r)}\right)\right) \hat{\partial}^{-1} Q^{(r)}-\left(P^{(r)} \hat{\partial}^{-1} Q^{(r)} \hat{\partial}^{n}\right)_{-} .
$$

Notice that the second term can be rewritten in the following way

$$
\begin{array}{r}
\left(P^{(r)} \hat{\partial}^{-1} Q^{(r)} \hat{\partial}^{n}\right)_{-}=\left(P^{(r)} Q^{(r)} \hat{\partial}^{n-1}-P^{(r)} \hat{\partial}^{-1}\left(\hat{\partial} Q^{(r)}\right) \hat{\partial}^{n-1}\right)_{-} \\
=\left(P^{(r)} \hat{\partial}^{-1}\left(-\hat{\partial} Q^{(r)}\right) \hat{\partial}^{n-1}\right)_{-}=\cdots=P^{(r)} \hat{\partial}^{-1}\left((-\hat{\partial})^{n}\left(Q^{(r)}\right)\right)=P^{(r)} \hat{\partial}^{-1}\left(\hat{\partial}^{n}\right)^{*}\left(Q^{(r)}\right),
\end{array}
$$

then the lemma is proved.

We can get some properties of the Lax operator in the following proposition.

Lemma 3.2. The Lax operator $\mathcal{L}$ of the constrained $N$-component KP hierarchy will satisfy the relation of

$$
\left(\mathcal{L}^{k}\right)_{-}=\sum_{j=0}^{k-1} \mathcal{L}^{k-j-1}\left(P^{(r)}\right) \hat{\partial}^{-1}\left(\mathcal{L}^{*}\right)^{j}\left(Q^{(r)}\right), \quad k \in \mathbb{Z} .
$$

where $\mathcal{L}(\phi):=(\mathcal{L})_{+}(\phi)+P^{(r)} \hat{\partial}^{-1}\left(Q^{(r)} \phi\right)$, for arbitrary $r \times r$ matrix function $\phi$.

The action of original additional flows of the constrained $N$-component KP hierarchy is expressed by

$$
\left(\partial_{1, k, \beta} \mathcal{L}\right)_{-}=\left[\left(\mathcal{M} C_{k}^{(\beta, r)}\right)_{+}, \mathcal{L}\right]_{-}+\left(C_{k}^{(\beta, r)}\right)_{-} .
$$

To keep the consistency with flow equations on eigenfunction and adjoint eigenfunction $P^{(r)}, Q^{(r)}$, we shall introduce an operator $T_{k}^{(\alpha, r)}$ as following to modify the additional symmetry of the constrained multi-component KP hierarchy.

We now introduce a pseudo differential operator $T_{k}^{(\alpha, r)}$,

$$
\begin{aligned}
T_{k}^{(\alpha, r)} & =0, k=-1,0,1, \\
T_{k}^{(\alpha, r)} & =\sum_{j=0}^{k-1}\left[j-\frac{1}{2}(k-1)\right] C_{k-1-j}^{(\alpha, r)}\left(P^{(r)}\right) \hat{\partial}^{-1}\left(C_{j}^{(\alpha, r) *}\right)\left(Q^{(r)}\right), k \geq 2 .
\end{aligned}
$$

The following lemmas are necessary to concern the Virasoro symmetry.

Lemma 3.3. If $X=M \hat{\partial}^{-1} N$, then

$$
[X, \mathcal{L}]_{-}=\left[M \hat{\partial}^{-1} \mathcal{L}^{*}(N)-\mathcal{L}(M) \hat{\partial}^{-1} N\right]+\left[X\left(P^{(r)}\right) \hat{\partial}^{-1} Q^{(r)}-P^{(r)} \hat{\partial}^{-1} X^{*}\left(Q^{(r)}\right)\right] .
$$

Lemma 3.4. The action of flows $\partial_{t_{l, \beta}}$ of the constrained $N$-component KP hierarchy on the $T_{k}^{(\alpha, r)}$ is

$$
\partial_{t_{l, \beta}} T_{k}^{(\alpha, r)}=\left[B_{l}^{(\beta, r)}, T_{k}^{(\alpha, r)}\right]_{-} .
$$


Proof.

$$
\begin{aligned}
\partial_{t_{l, \beta}} T_{k}^{(\alpha, r)}= & \partial_{t_{l, \beta}}\left(\sum_{j=0}^{k-1}\left[j-\frac{1}{2}(k-1)\right] C_{k-1-j}^{(\alpha, r)}\left(P^{(r)}\right) \hat{\partial}^{-1}\left(C_{j}^{(\alpha, r) *}\right)\left(Q^{(r)}\right)\right) \\
= & \sum_{j=0}^{k-1}\left[j-\frac{1}{2}(k-1)\right]\left\{\partial_{t_{l, \beta}}\left(C_{k-1-j}^{(\alpha, r)}\left(P^{(r)}\right)\right) \hat{\partial}^{-1}\left(C_{j}^{(\alpha, r) *}\right)\left(Q^{(r)}\right)\right. \\
& \left.+C_{k-1-j}^{(\alpha, r)}\left(P^{(r)}\right) \hat{\partial}^{-1} \partial_{t_{l, \beta}}\left(\left(C_{j}^{(\alpha, r) *}\right)\left(Q^{(r)}\right)\right)\right\} \\
= & {\left[B_{l}^{(\beta, r)} \circ \sum_{j=0}^{k-1}\left[j-\frac{1}{2}(k-1)\right] C_{k-1-j}^{(\alpha, r)}\left(P^{(r)}\right) \hat{\partial}^{-1}\left(C_{j}^{(\alpha, r) *}\right)\left(Q^{(r)}\right)\right]_{-} } \\
& -\left[\left(\sum_{j=0}^{k-1}\left[j-\frac{1}{2}(k-1)\right] C_{k-1-j}^{(\alpha, r)}\left(P^{(r)}\right) \hat{\partial}^{-1}\left(C_{j}^{(\alpha, r) *}\right)\left(Q^{(r)}\right)\right) \circ B_{l}^{(\beta, r)}\right]_{-} \\
= & {\left[B_{l}^{(\beta, r)},\left(\sum_{j=0}^{k-1}\left[j-\frac{1}{2}(k-1)\right] C_{k-1-j}^{(\alpha, r)}\left(P^{(r)}\right) \hat{\partial}^{-1}\left(C_{j}^{(\alpha, r) *}\right)\left(Q^{(r)}\right)\right)\right]_{-} } \\
= & {\left[B_{l}^{(\beta, r)}, T_{k}^{(\alpha, r)}\right]_{-} . }
\end{aligned}
$$

Further, the following expression of $\left[T_{k-1}^{(\beta, r)}, \mathcal{L}\right]_{-}$is also necessary to define the additional flows of the constrained $N$-component KP hierarchy.

Lemma 3.5. The Lax operator $\mathcal{L}$ of the constrained $N$-component KP hierarchy and $T_{k-1}^{(\beta, r)}$ has the following relation,

$$
\begin{aligned}
{\left[T_{k-1}^{(\beta, r)}, \mathcal{L}\right]_{-}=} & -\left(C_{k}^{(\beta, r)}\right)_{-}+\frac{k}{2}\left[P^{(r)} \hat{\partial}^{-1}\left(C_{k-1}^{(\beta, r)}\right)^{*}\left(Q^{(r)}\right)+C_{k-1}^{(\beta, r)}\left(P^{(r)}\right) \hat{\partial}^{-1} Q^{(r)}\right. \\
& \left.+T_{k-1}^{(\beta, r)}\left(P^{(r)}\right) \hat{\partial}^{-1} Q^{(r)}-P^{(r)} \hat{\partial}^{-1}\left(T_{k-1}^{(\beta, r)}\right)^{*}\left(Q^{(r)}\right)\right]
\end{aligned}
$$

Proof. A direct calculation can lead to

$$
\begin{aligned}
{\left[T_{k-1}^{(\beta, r)}, \mathcal{L}\right]_{-}=} & {\left[\sum_{j=0}^{k-2}\left[j-\frac{1}{2}(k-2)\right] C_{k-2-j}^{(\beta, r)}\left(P^{(r)}\right) \hat{\partial}^{-1}\left(C_{j}^{(\beta, r)}\right)^{*}\left(Q^{(r)}\right), \mathcal{L}\right]_{-} } \\
= & \sum_{j=0}^{k-2}\left[j-\frac{1}{2}(k-2)\right] C_{k-2-j}^{(\beta, r)}\left(P^{(r)}\right) \hat{\partial}^{-1}\left(C_{j+1}^{(\beta, r)}\right)^{*}\left(Q^{(r)}\right) \\
& -\sum_{j=0}^{k-2}\left[j-\frac{1}{2}(k-2)\right] C_{k-1-j}^{(\alpha, r)}\left(P^{(r)}\right) \hat{\partial}^{-1}\left(C_{j}^{(\beta, r)}\right)^{*}\left(Q^{(r)}\right) \\
& +\left(T_{k-1}^{(\beta, r)}\left(P^{(r)}\right) \hat{\partial}^{-1} Q^{(r)}-P^{(r)} \hat{\partial}^{-1}\left(T_{k-1}^{(\beta, r)}\right)^{*}\left(Q^{(r)}\right)\right)
\end{aligned}
$$




$$
\begin{aligned}
= & -\sum_{j=1}^{k-2} C_{k-1-j}^{(\beta, r)}\left(P^{(r)}\right) \hat{\partial}^{-1}\left(C_{j}^{(\beta, r)}\right)^{*}\left(Q^{(r)}\right) \\
& +\left(\frac{k}{2}-1\right)\left[P^{(r)} \hat{\partial}^{-1}\left(C_{k-1}^{(\beta, r)^{*}}\left(Q^{(r)}\right)+C_{k-1}^{(\beta, r)}\left(P^{(r)}\right) \hat{\partial}^{-1} Q^{(r)}\right]\right. \\
& +\left(T_{k-1}^{(\beta, r)}\left(P^{(r)}\right) \hat{\partial}^{-1} Q^{(r)}-P^{(r)} \hat{\partial}^{-1}\left(T_{k-1}^{(\beta, r)}\right)^{*}\left(Q^{(r)}\right)\right),
\end{aligned}
$$

which further help us deriving eq.(3.16) using eq.(3.10).

Putting together (3.11) and (3.16), we define the additional flows of the constrained $N$ component KP hierarchy as

$$
\partial_{t_{1, k, \beta}} \mathcal{L}=\left[-\left(\mathcal{M} C_{k}^{(\beta, r)}\right)_{-}+T_{k-1}^{(\beta, r)}, \mathcal{L}\right],
$$

where $T_{k-1}^{(\beta, r)}=0$, for $k=0,1,2$, such that the right-hand side of (3.17) is in the form of derivation of Lax equations. Generally, one can also derive

$$
\partial_{t_{1, k, \beta}}\left(\mathcal{M} \mathcal{L}^{l}\right)=\left[-\left(\mathcal{M} C_{k}^{(\beta, r)}\right)_{-}+T_{k-1}^{(\beta, r)}, \mathcal{M L}^{l}\right]
$$

Now we calculate the action of the additional flows eq.(3.17) on the eigenfunction $P^{(r)}$ and $Q^{(r)}$ of the constrained $N$-component KP hierarchy.

Theorem 3.6. The acting of additional flows of constrained $N$-component KP hierarchy on the eigenfunction $P^{(r)}$ and $Q^{(r)}$ are

$$
\begin{aligned}
& \partial_{t_{1, k, \beta}} P^{(r)}=\left(\mathcal{M} C_{k}^{(\beta, r)}\right)_{+}\left(P^{(r)}\right)+T_{k-1}^{(\beta, r)}\left(P^{(r)}\right)+\frac{k}{2} C_{k-1}^{(\beta, r)}\left(P^{(r)}\right), \\
& \partial_{t_{1, k, \beta}} Q^{(r)}=-\left(\mathcal{M} C_{k}^{(\beta, r)}\right)_{+}^{*}\left(Q^{(r)}\right)-\left(T_{k-1}^{(\beta, r)}\right)^{*}\left(Q^{(r)}\right)+\frac{k}{2} C_{k-1}^{(\beta, r) *}\left(Q^{(r)}\right) .
\end{aligned}
$$

Proof. Substitution of (3.16) to negative part of (3.17) shows

$$
\begin{aligned}
\left(\partial_{t_{1, k, \beta}} \mathcal{L}\right)_{-} & =\left(\mathcal{M} C_{k}^{(\beta, r)}\right)_{+}\left(P^{(r)}\right) \hat{\partial}^{-1}\left(Q^{(r)}\right)-P^{(r)} \hat{\partial}^{-1}\left(\mathcal{M} C_{k}^{(\beta, r)}\right)_{+}^{*}\left(Q^{(r)}\right)+T_{k-1}^{(\beta, r)}\left(P^{(r)}\right) \hat{\partial}^{-1} Q^{(r)} \\
& -P^{(r)} \hat{\partial}^{-1}\left(T_{k-1}^{(\beta, r)}\right)^{*}\left(Q^{(r)}\right)+\frac{k}{2} P^{(r)} \hat{\partial}^{-1}\left(T_{k-1}^{(\beta, r)}\right)^{*}\left(Q^{(r)}\right)+\frac{k}{2} C_{k}^{(\beta, r)}\left(P^{(r)}\right) \hat{\partial}^{-1} Q^{(r)}
\end{aligned}
$$

On the other side,

$$
\left(\partial_{t_{1, k, \beta}} \mathcal{L}\right)_{-}=\left(\partial_{t_{1, k, \beta}} P^{(r)}\right) \hat{\partial}^{-1} Q^{(r)}+P^{(r)} \hat{\partial}^{-1}\left(\partial_{t_{1, k, \beta}} Q^{(r)}\right) .
$$

Comparing right hand sides of (3.20) and (3.21) implies the action of additional flows on the eigenfunction and the adjoint eigenfunction (3.19).

Next we shall prove the commutation relation between the additional flows $\partial_{t_{1, k, \beta}}$ of constrained $N$-component KP hierarchy and the original flows $\partial_{t_{l, \alpha}}$ of it.

Theorem 3.7. The additional flows of $\partial_{t_{1, k, \beta}}$ are symmetry flows of the constrained $N$-component $K P$ hierarchy, i.e. they commute with all $\partial_{t_{l, \alpha}}$ flows of the constrained $N$-component KP hierarchy. 
Proof. According the action of $\partial_{t_{1, k, \beta}}$ and $\partial_{t_{l, \alpha}}$ on the dressing operator $S$, then

$$
\begin{aligned}
{\left[\partial_{t_{1, k, \beta}}, \partial_{t_{l, \alpha}}\right] S=} & -\partial_{t_{1, k, \beta}}\left(\left(C_{l}^{(\alpha, r)}\right)_{-} S\right)-\partial_{t_{l, \alpha}}\left[-\left(\mathcal{M} C_{k}^{(\beta, r)}\right)_{-}+T_{k-1}^{(\beta, r)}\right] S \\
= & \left(-\partial_{t_{1, k, \beta}} C_{l}^{(\alpha, r)}\right)_{-} S-\left(C_{l}^{(\beta, r)}\right)_{-} \partial_{t_{1, k, \beta}} S-\left[\left(\mathcal{M} C_{k}^{(\beta, r)}\right)_{-}-T_{k-1}^{(\beta, r)}\right]\left(C_{l}^{(\alpha, r)}\right)_{-} S \\
& +\left[\left(C_{l}^{(\alpha, r)}\right)_{+}, \mathcal{M} C_{k}^{(\beta, r)}\right]_{-} S-\left(\partial_{t_{l, \alpha}} T_{k-1}^{(\beta, r)}\right) S \\
= & {\left[\left(C_{l}^{(\alpha, r)}\right)_{-},-T_{k-1}^{(\beta, r)}\right]_{-} S+\left[-T_{k-1}^{(\beta, r)}, C_{l}^{(\alpha, r)}\right]_{-} S-\left(\partial_{t_{l, \alpha}} T_{k-1}^{(\beta, r)}\right) S } \\
= & {\left[B_{l}^{(\alpha, r)}, T_{k-1}^{(\beta, r)}\right]_{-} S-\left(\partial_{t_{1, \alpha}} T_{k-1}^{(\beta, r)}\right) S } \\
= & 0 .
\end{aligned}
$$

Therefore the theorem holds.

Taking into account $T_{k-1}^{(\beta, r)}=0$ for $k=0,1,2$, then eq.(3.19) becomes

$$
\begin{aligned}
& \partial_{t_{1, l, \beta}} P^{(r)}=\left(\mathcal{M} C_{l}^{(\beta, r)}\right)_{+}\left(P^{(r)}\right)+\frac{1}{2} l C_{l-1}^{(\beta, r)} P^{(r)}, \quad l=0,1,2, \\
& \partial_{t_{1, l, \beta}} Q^{(r)}=-\left(\mathcal{M} C_{l}^{(\beta, r)}\right)_{+}^{*}\left(Q^{(r)}\right)+\frac{1}{2} l C_{l-1}^{(\beta, r)^{*}} Q^{(r)}, \quad l=0,1,2 .
\end{aligned}
$$

Then using eq. (3.22) and the relation $\partial_{t_{1, l, \beta}}\left(\mathcal{L}^{k}\left(P^{(r)}\right)\right)=\left(\partial_{t_{1, l, \beta}}\left(\mathcal{L}^{k}\right)\right)\left(P^{(r)}\right)+\mathcal{L}^{k} \partial_{t_{1, l, \beta}}\left(P^{(r)}\right)$, we can find the additional flows $\partial_{t_{1, l, \beta}}$ of constrained $N$-component KP hierarchy have the following relations

$$
\begin{aligned}
\partial_{t_{1, l, \beta}} C_{k}^{(\alpha, r)}\left(P^{(r)}\right) & =\left(\mathcal{M} C_{l}^{(\beta, r)}\right)_{+}\left(C_{k}^{(\alpha, r)}\left(P^{(r)}\right)\right)+\left(k+\frac{l}{2}\right) C_{k+l-1}^{(\alpha, r)} \delta_{\alpha, \beta}\left(P^{(r)}\right)+T_{l-1}^{(\beta, r)} C_{k}^{(\alpha, r)}\left(P^{(r)}\right) \\
\partial_{t_{1, l, \beta}} C_{k}^{(\alpha, r)^{*}}\left(Q^{(r)}\right) & =-\left(\mathcal{M} C_{l}^{(\beta, r)}\right)_{+}^{*} C_{k}^{(\alpha, r)^{*}}\left(Q^{(r)}\right)+\left(k+\frac{l}{2}\right) C_{k+l-1}^{(\alpha, r)}{ }^{*} \delta_{\alpha, \beta}\left(Q^{(r)}\right)+\left(T_{l-1}^{(\beta, r)} C_{k}^{(\alpha, r)}\right)^{*}\left(P^{(r)}\right)
\end{aligned}
$$

Moreover, the action of $\partial_{t_{1, l, \beta}}$ on $T_{k}^{(\alpha, r)}$ is given by the following lemma.

Lemma 3.8. The actions on $T_{k}^{(\alpha, r)}$ of the additional symmetry flows $\partial_{t_{1, l, \beta}}$ of the constrained $N$-component KP hierarchy are

$$
\partial_{t_{1, l, \beta}} T_{k}^{(\alpha, r)}=\left[\left(\mathcal{M} C_{l}^{\beta, r}\right)_{+}+T_{l-1}^{(\beta, r)}, T_{k}^{(\alpha, r)}\right]_{-}+(k-l+1) T_{k+l-1}^{(\alpha, r)} \delta_{\alpha, \beta}
$$


Proof. Using eq.(3.23), a straightforward calculation implies

$$
\begin{aligned}
\partial_{t_{1, l, \beta}} T_{k}^{(\alpha, r)} & =\partial_{t_{1, l, \beta}}\left(\sum_{j=0}^{k-1}\left[j-\frac{1}{2}(k-1)\right] C_{k-1-j}^{(\alpha, r)}\left(P^{(r)}\right) \hat{\partial}^{-1}\left(C_{j}^{(\alpha, r) *}\right)\left(Q^{(r)}\right)\right) \\
& =\sum_{j=0}^{k-1}\left[j-\frac{1}{2}(k-1)\right]\left(\partial_{t_{1, l, \beta}}\left(C_{k-1-j}^{(\alpha, r)}\left(P^{(r)}\right)\right) \hat{\partial}^{-1}\left(C_{j}^{(\alpha, r) *}\right)\left(Q^{(r)}\right)\right. \\
& \left.+C_{k-1-j}^{(\alpha, r)}\left(P^{(r)}\right) \hat{\partial}^{-1}\left(\partial_{t_{1, l, \beta}}\left(C_{j}^{(\alpha, r) *}\right)\left(Q^{(r)}\right)\right)\right) \\
& =\sum_{j=0}^{k-1}\left[j-\frac{1}{2}(k-1)\right]\left[\left(\mathcal{M} C_{l}^{\beta, r}\right)_{+}+T_{l-1}^{(\beta, r)}\right]\left(C_{k-1-j}^{(\alpha, r)}\left(P^{(r)}\right)\right) \hat{\partial}^{-1}\left(C_{l}^{\beta, r}\right)^{*}\left(Q^{(r)}\right) \\
& +\sum_{j=0}^{k-1}\left[j-\frac{1}{2}(k-1)\right]\left(k-j-1+\frac{l}{2}\right) C_{k+l-2-j}^{\alpha, r}\left(P^{(r)}\right) \hat{\partial}^{-1}\left(C_{j}^{\beta, r}\right)^{*}\left(Q^{(r)}\right) \delta_{\alpha, \beta} \\
& -\sum_{j=0}^{k-1}\left[j-\frac{1}{2}(k-1)\right] C_{k-1-j}^{(\alpha, r)}\left(P^{(r)}\right) \hat{\partial}^{-1}\left[\left(\mathcal{M} C_{l}^{\beta, r}\right)_{+}^{*}+\left(T_{l-1}^{(\beta, r)}\right)^{*}\right]\left(C_{j}^{\beta, r}\right)^{*}\left(Q^{(r)}\right) \\
& +\sum_{j=0}^{k-1}\left[j-\frac{1}{2}(k-1)\right]\left(j+\frac{l}{2}\right) C_{k-1-j}^{(\alpha, r)}\left(P^{(r)}\right) \hat{\partial}^{-1}\left(C_{j+l-1}^{\beta, r}\right)^{*}\left(Q^{(r)}\right) \delta_{\alpha, \beta},
\end{aligned}
$$

which can be simplified to eq.(3.24).

Now it is time to identity the algebraic structure of the additional symmetry flows of the constrained $N$-component KP hierarchy.

Theorem 3.9. The additional flows $\partial_{t_{1, k, \beta}}$ of the constrained $N$-component KP hierarchy form the positive half of Virasoro algebra, i.e., for $l=0,1,2 ; k \geq 0,1 \leq \alpha, \beta \leq r$,

$$
\left[\partial_{t_{1, l, \alpha}}, \partial_{t_{1, k, \beta}}\right]=(k-l) \delta_{\alpha, \beta} \partial_{t_{1, k+l-1, \alpha}} .
$$

Proof. Using $\left[\left(\mathcal{M} C_{l}^{(\alpha, r)}\right)_{-}, T_{k-1}^{(\beta, r)}\right]_{-}=\left[\left(\mathcal{M} C_{l}^{(\alpha, r)}\right)_{-}, T_{k-1}^{(\beta, r)}\right]$ and the Jacobi identity, one can derive the following computation

$$
\begin{aligned}
& {\left[\partial_{t_{1, l, \alpha}}, \partial_{t_{1, k, \beta}}\right] \mathcal{L} } \\
= & \left.\partial_{t_{1, l, \alpha}}\left(\left[-\left(\mathcal{M} C_{k}^{(\beta, r)}\right)_{-}, \mathcal{L}\right]+\left[T_{k-1}^{\beta, r}, \mathcal{L}\right]\right)-\partial_{t_{1, k, \beta}}\left(\left[-\left(\mathcal{M} C_{l}^{\alpha, r}\right)_{-}, \mathcal{L}\right]+\left[T_{l-1}^{\alpha, r}, \mathcal{L}\right]\right)\right) \\
= & \partial_{t_{1, l, \alpha}}\left[-\left(\mathcal{M} C_{k}^{(\beta, r)}\right)_{-}, \mathcal{L}\right]+\left[\partial_{t_{1, l, \alpha}} T_{k-1}^{\beta, r}, \mathcal{L}\right]+\left[T_{k-1}^{\beta, r}, \partial_{t_{1, l, \alpha}} \mathcal{L}\right]+\left[\partial_{t_{1, k, \beta}}\left(\mathcal{M} C_{l}^{\alpha, r}\right)_{-}, \mathcal{L}\right] \\
& +\left[\left(\mathcal{M} C_{l}^{\alpha, r}\right)_{-}, \partial_{t_{1, k, \beta}} \mathcal{L}\right]-\left[\partial_{t_{1, k, \beta}} T_{l-1}^{\alpha, r}, \mathcal{L}\right]-\left[T_{l-1}^{\alpha, r}, \partial_{t_{1, k, \beta}} \mathcal{L}\right] \\
= & {\left[\left[\left(\mathcal{M} C_{l}^{(\alpha, r)}\right)_{-}, \mathcal{M} C_{k}^{\beta, r}\right]_{-}, \mathcal{L}\right]+\left[-\left(\mathcal{M} C_{k}^{(\beta, r)}\right)_{-},\left[-\left(\mathcal{M} C_{l}^{(\alpha, r)}\right)_{-}, \mathcal{L}\right]\right] } \\
+ & {\left[\left[\left(\mathcal{M} C_{l}^{\alpha, r}\right)_{+}, T_{k-1}^{\beta, r}\right]_{-}+(k-l) T_{k+l-2}^{(\beta, r)} \delta_{\alpha, \beta}, \mathcal{L}\right] }
\end{aligned}
$$




$$
\begin{aligned}
+ & {\left[T_{k-1}^{\beta, r},\left[-\left(\mathcal{M} C_{l}^{\alpha, r}\right)_{-}, \mathcal{L}\right]\right]+\left[\left[-\left(\mathcal{M} C_{k}^{(\beta, r)}\right)_{-}+T_{k-1}^{\beta, r}, \mathcal{M} C_{l}^{\alpha, r}\right]_{-}, \mathcal{L}\right] } \\
+ & {\left[\left(\mathcal{M} C_{l}^{\alpha, r}\right)_{-},\left[-\left(\mathcal{M} C_{k}^{(\beta, r)}\right)_{-}+T_{k-1}^{\beta, r}, \mathcal{L}\right]\right] } \\
= & {\left[\left(\left(\mathcal{M} C_{l}^{(\alpha, r)}\right)_{-}, \mathcal{M} C_{k}^{\beta, r}\right]_{-}, \mathcal{L}\right]+\left[-\left(\mathcal{M} C_{k}^{(\beta, r)}\right)_{-},\left[-\left(\mathcal{M} C_{l}^{(\alpha, r)}\right)_{-}, \mathcal{L}\right]\right] } \\
+ & {\left[(k-l) T_{k+l-2}^{(\beta, r)} \delta_{\alpha, \beta}, \mathcal{L}\right]+\left[\left[-\left(\mathcal{M} C_{k}^{(\beta, r)}\right)_{-}, \mathcal{M} C_{l}^{\alpha, r}\right]_{-}, \mathcal{L}\right] } \\
& +\left[\left(\mathcal{M} C_{l}^{\alpha, r}\right)_{-},\left[-\left(\mathcal{M} C_{k}^{(\beta, r)}\right)_{-}, \mathcal{L}\right]\right] \\
= & (k-l)\left[-\delta_{\alpha, \beta}\left(\mathcal{M} C_{l+k-1}^{(\alpha, r)}\right)_{-}, \mathcal{L}\right]+\left[(k-l) T_{k+l-2}^{(\beta, r)} \delta_{\alpha, \beta}, \mathcal{L}\right] \\
= & (k-l) \delta_{\alpha, \beta} \partial_{t_{1, k+l-1, \alpha}} \mathcal{L} .
\end{aligned}
$$

We define $L_{k-1, \alpha}:=\partial_{t_{1, k, \alpha}}$, and obtain recursively all higher Virasoro operators as

$$
L_{k+1, \alpha} \delta_{\alpha, \beta}=-\frac{1}{k-1}\left[L_{k, \alpha}, L_{1, \beta}\right], \quad k \geq 2
$$

By induction as

$$
\left[L_{m, \alpha}, L_{k+1, \beta}\right]=\frac{m-1}{k-1}\left[L_{k, \beta}, L_{m+1, \alpha}\right]+\frac{k-m}{k-1}\left[L_{1, \alpha}, L_{m+k, \beta}\right],
$$

the obtained higher Virasoro operators in the above recursive way form a closed matrix-formed Virasoro algebra up to irrelevant terms containing flows which commute with the $\operatorname{sl}(2)$ additional symmetry generators $\left\{L_{-1, \alpha}, L_{0, \alpha}, L_{1, \alpha} ; 1 \leq \alpha \leq r\right\}$.

\section{The Discrete CONSTRAined MULTi-COMPONENT KP HiERARCHY}

At the beginning of the this section, to make this paper be self-contained, let us firstly recall some useful facts of discrete calculus.

For any function $f(n)$, the discrete differential $\Delta$ is defined by

$$
\Delta(f(n))=f(n+1)-f(n),
$$

and the discrete shift operator $\Lambda$ is defined by $\Lambda(f(n))=f(n+1)$. We denote the formal inverse of $\Delta$ as $\Delta^{-1}$. The following discrete deformed Leibnitz rule holds

$$
\Delta^{n} \circ f=\sum_{k \geq 0}\left(\begin{array}{l}
n \\
k
\end{array}\right) \Lambda^{n-k}\left(\Delta^{k} f\right) \Delta^{n-k}, \quad n \in \mathbb{Z} .
$$

For any two discrete pseudo-differential operators $W=\sum_{i} p_{i}(n) \Delta^{i}$, the conjugate operation “*” for $W$ is defined by $W^{*}=\sum_{i}\left(\Delta^{*}\right)^{i} p_{i}(n)$ with

$$
\Delta^{*}=-\Delta \Lambda^{-1}, \quad\left(\Delta^{-1}\right)^{*}=\left(\Delta^{*}\right)^{-1}=-\Lambda \Delta^{-1}
$$

Now we prove some useful properties for the operators which are used later.

Lemma 2.1 For arbitrary function $f$ and $\Delta, \Lambda$ as above, the following identities hold.

(1) $\Delta \circ \Lambda=\Lambda \circ \Delta$, 


$$
\begin{aligned}
& \text { (2) } \Delta^{-1} \circ f \circ \Delta^{-1}=\left(\Delta^{-1} f\right) \circ \Delta^{-1}-\Delta^{-1} \circ \Lambda\left(\Delta^{-1} f\right), \\
& \text { (3) } \Delta^{-1} \circ f=\Lambda^{-1}(f) \circ \Delta^{-1}+\Delta^{-1} \circ\left(\Delta^{*} f\right) \circ \Delta^{-1} .
\end{aligned}
$$

Proof. The proof is standard and direct. We omit it here.

Because of the above lemma, for arbitrary function $f_{1}, g_{1}, f_{2}, g_{2}$, the following formula can be derived

$$
f_{1} \Delta^{-1} g_{1} \circ f_{2} \Delta^{-1} g_{2}=f_{1} \Delta^{-1}\left(g_{1}, f_{2}\right) \Delta^{-1} g_{2}-f_{1} \Delta^{-1} \circ \Lambda\left(\Delta^{-1}\left(g_{1}, f_{2}\right)\right) g_{2} .
$$

Proposition 4.1. The following identities hold:

$$
\begin{aligned}
\operatorname{res}((\Delta A)(n)) & =\operatorname{res}(\Delta \circ A(n)-A(n+1) \circ \Delta), \\
P_{<0}\left(\Delta^{-1} A\right) & =\Delta^{-1} P_{<0}(A)+\Delta^{-1} P_{0}\left(A^{*}\right), \\
\operatorname{res}\left(\Delta^{-1} A\right) & =\left(\Lambda^{-1} P_{0}\left(A^{*}\right)\right),
\end{aligned}
$$

where $(\Delta A)$ denotes the action of $\Delta$ on operator $A,\left(\Lambda^{-1} P_{0}\left(A^{*}\right)\right)$ means a backward shift of function $P_{0}\left(A^{*}\right)$ (zero order term of operator $A^{*}$ on $\Delta$ ) on $x$.

Proof. One can check the proof in [8].

After integrable discretization by $u(n)=u(x+n \epsilon)$, one can get similar results as constrained multi-component KP hierarchy for its discrete version.

Using the above proposition, the following proposition can be easily got similarly as [8].

Proposition 4.2. If $\alpha$ and $\beta$ are two local discrete operators, then

$$
\operatorname{res}\left(\Delta^{-1} \alpha \beta \Delta^{-1}\right)=\operatorname{res}\left(\Delta^{-1} P_{0}\left(\alpha^{*}\right) \beta \Delta^{-1}\right)+\operatorname{res}\left(\Delta^{-1} \alpha P_{0}(\beta) \Delta^{-1}\right) .
$$

Proof. See the proof in [8].

The Lax operator $L_{\Delta}$ of discrete multicomponent KP hierarchy is given by

$$
L_{\Delta}=E \Delta+v_{0}+v_{-1} \Delta^{-1}+v_{-2} \Delta^{-2}+\cdots .
$$

where $v_{i}=v_{i}\left(n, t_{\alpha, n}, \cdots,\right), i=0,-1,-2,-3, \cdots$ are $N \times N$ matrices.

There are another $N$ pseudo difference operators $R_{\Delta 1}, \ldots, R_{\Delta N}$ of the form

$$
R_{\Delta \alpha}=E_{\alpha}+v_{\alpha 1} \Delta^{-1}+v_{\alpha 2} \Delta^{-2}+\ldots,
$$

where $E_{\alpha}$ is the $N \times N$ matrix with 1 on the $(\alpha, \alpha)$-component and zero elsewhere, and $v_{\alpha 1}, v_{\alpha 2}, \ldots$ are also $N \times N$ matrices. The operators $L_{\Delta}, R_{\Delta 1}, \ldots, R_{\Delta N}$ satisfy the following conditions:

$$
L_{\Delta} R_{\Delta \alpha}=R_{\Delta \alpha} L_{\Delta}, \quad R_{\Delta \alpha} R_{\Delta \beta}=\delta_{\alpha \beta} R_{\Delta \alpha}, \quad \sum_{\alpha=1}^{N} R_{\Delta \alpha}=E .
$$

The Lax equations of the discrete multi-component KP (dmKP) hierarchy are:

$$
\frac{\partial L_{\Delta}}{\partial t_{\alpha, n}}=\left[B_{\Delta \alpha, n}, L_{\Delta}\right], \quad \frac{\partial R_{\Delta \beta}}{\partial t_{\alpha, n}}=\left[B_{\Delta \alpha, n}, R_{\Delta \beta}\right], \quad B_{\Delta \alpha, n}:=\left(L_{\Delta}^{n} R_{\Delta \alpha}\right)_{+} .
$$


In fact the Lax operator $L_{\Delta}$ in eq. (4.11) and $R_{\Delta \alpha}$ can have the following dressing structures

$$
L_{\Delta}=S_{\Delta} \Delta S_{\Delta}^{-1}, \quad R_{\Delta \alpha}=S_{\Delta} E_{\alpha} S_{\Delta}^{-1}
$$

where

$$
S_{\Delta}=E+\sum_{i=1}^{\infty} S_{\Delta i} \Delta^{-i}
$$

Dressing operator $S_{\Delta}$ satisfies Sato equation

$$
\frac{\partial S_{\Delta}}{\partial t_{\alpha, n}}=-\left(L_{\Delta}^{n} R_{\Delta \alpha}\right)_{-} S_{\Delta}, \quad 1 \leq \alpha \leq N, n=1,2,3, \cdots
$$

Considering one kind of reduction of the discrete multi-component KP hierarchy, the hierarchy will become the discrete constrained multi-component KP hierarchy in the next subsection.

As [13], the following reduction condition is imposed onto the discrete $N$-component KP hierarchy:

$$
\sum_{l=1}^{r} d_{l}^{s}\left(L_{\Delta} R_{\Delta l}\right)^{s}+\sum_{l=r+1}^{N} L_{\Delta} R_{\Delta l}=\sum_{l=1}^{r} d_{l}^{s}\left(L_{\Delta} R_{\Delta l}\right)_{+}^{s}+\sum_{l=r+1}^{N}\left(L_{\Delta} R_{\Delta l}\right)_{+}, \quad 1 \leq r \leq N .
$$

Let us denote following operators as [13]

$$
\begin{gathered}
\hat{\Delta}:=\partial_{1}+\partial_{1}+\cdots+\partial_{r}, \quad \partial_{i}=\frac{\partial}{\partial t_{i, 1}} \\
\hat{L}_{\Delta}^{(i)}=S_{\Delta}^{(r)}(\hat{\Delta}) E_{i}^{(r)} \hat{\Delta}\left(S_{\Delta}^{(r)}(\hat{\Delta})\right)^{-1}, \quad \hat{L}_{\Delta}=\sum_{i=1}^{r} d_{i} \hat{L}_{\Delta}^{(i)},
\end{gathered}
$$

and

$$
\begin{gathered}
B_{\Delta k}^{(l, r)}=\left(S_{\Delta}^{(r)}(\hat{\Delta}) E_{l}^{(r)} \hat{\Delta}^{k}\left(S_{\Delta}^{(r)}(\hat{\Delta})\right)^{-1}\right)_{+}=\left(C_{\Delta k}^{(l, r)}\right)_{+}, \quad 1 \leq l \leq r, \\
\phi_{\Delta}^{(r)}=\left.\left(S_{\Delta 1}\right)_{i j}\right|_{1 \leq i \leq r, r+1 \leq j \leq N}, \quad \psi_{\Delta}^{(r)}=\left.\left(S_{\Delta 1}\right)_{i j}\right|_{1 \leq j \leq r, r+1 \leq i \leq N},
\end{gathered}
$$

where $S_{\Delta}^{(r)}$ is the $r \times r$ principal sub-matrix of $S_{\Delta}$ and the projections here is about the operator $\hat{\Delta}$. This further lead to

$$
\mathcal{L}_{\Delta}=\hat{L}_{\Delta}^{s}=\sum_{l=1}^{r} d_{l}^{s} B_{\Delta s}^{(l, r)}+\phi^{(r)} \hat{\Delta}^{-1} \psi^{(r)}
$$

Under the constraint eq.(4.15), the following evolutionary equations of the discrete constrained $N$-component KP hierarchy can be derived.

Proposition 4.3. For discrete constrained $N$-component KP hierarchy, the following hierarchies of equations can be derived [13] 


$$
\begin{gathered}
\frac{\partial \phi^{(r)}}{\partial t_{n, l}}=B_{\Delta n}^{(l, r)} \phi^{(r)}, \quad \frac{\partial \psi^{(r)}}{\partial t_{n, l}}=-\left(B_{\Delta n}^{(l, r)}\right)^{*} \psi^{(r)} \\
\frac{\partial \mathcal{L}_{\Delta}}{\partial t_{n, l}}=\left[B_{\Delta n}^{(l, r)}, \mathcal{L}_{\Delta}\right]
\end{gathered}
$$

where for $B_{\Delta n}^{(l, r)}=\sum_{i=0}^{n} B_{i} \hat{\Delta}^{i}$,

$$
\left(B_{\Delta n}^{(l, r)}\right)^{*} \psi^{(r)}:=\sum_{i=0}^{n}(-1)^{i} \hat{\Delta}^{i} \Lambda^{-i}\left(\psi^{(r)} B_{i}\right) .
$$

When $r=1, N=2$, one can obtain the discrete $s$-constrained KP hierarchy. When $r=$ $1, N \geq 2$, one can call it the discrete vector $s$-constrained KP hierarchy. For general $r, N$, it is about the generalized discrete constrained KP hierarchy.

\section{VirAsoro SYMMETRY OF THE DISCRETE CONSTRAINED MULTI-COMPONENT KP HIERARCHY}

In this section, we shall construct the additional symmetry and discuss the algebraic structure of the additional symmetry flows of the multi-component discrete constrained KP hierarchy.

To this end, firstly we define $\hat{\Gamma}_{\Delta}^{(r)}$ and the Orlov-Shulman's operator $\mathcal{M}_{\Delta}$ as

$$
\hat{\Gamma}_{\Delta}^{(r)}=\sum_{\beta=1}^{r} t_{1 \beta} \frac{E_{\beta}}{s d_{\beta}^{s}} \hat{\Delta}^{1-s}+\sum_{\beta=1}^{r} \sum_{n=2}^{\infty} \frac{n}{s} d_{\beta}^{-s} E_{\beta} \hat{\Delta}^{n-s} t_{n \beta}, \quad \mathcal{M}_{\Delta}=S^{(r)} \hat{\Gamma}_{\Delta}^{(r)}\left(S^{(r)}\right)^{-1} .
$$

It is easy to find the following formula

$$
\left[\partial_{t_{k, \beta}}-\hat{\Delta}^{k} E_{\beta}, \hat{\Gamma}_{\Delta}^{(r)}\right]=0
$$

Define another operator

$$
\mathcal{M}_{\Delta}=W_{\Delta} \circ \hat{\Gamma}_{\Delta}^{(r)} \circ W_{\Delta}^{-1} .
$$

There are the following commutation relations

$$
\left[\sum_{\beta=1}^{r} d_{\beta}^{s} \hat{\Delta}^{s} E_{\beta}, \hat{\Gamma}_{\Delta}^{(r)}\right]=1,\left[\mathcal{L}_{\Delta}, \mathcal{M}_{\Delta}\right]=1
$$

which can be verified by a straightforward calculation. By using the Sato equation, the isospectral flow of the $\mathcal{M}_{\Delta}$ operator is given by

$$
\partial_{t_{k, \beta}} \mathcal{M}_{\Delta}=\left[\left(C_{\Delta k}^{\beta r}\right)_{+}, \mathcal{M}_{\Delta}\right]
$$

More generally,

$$
\partial_{t_{k, \beta}}\left(\mathcal{M}_{\Delta}^{m} \mathcal{L}_{\Delta}^{l}\right)=\left[\left(C_{\Delta k}^{\beta r}\right)_{+}, \mathcal{M}_{\Delta}^{m} \mathcal{L}_{\Delta}^{l}\right] .
$$


Based on the above preparation, the additional symmetry flows of the discrete constrained multi-component KP hierarchy are define by their actions on the dressing operator

$$
\partial_{l, m, \beta} S_{\Delta}=-\left(\mathcal{M}_{\Delta}^{m} C_{\Delta l}^{\beta r}\right)_{-} \circ S_{\Delta},
$$

or equivalently on the Lax operator

$$
\partial_{l, m, \beta} \mathcal{L}_{\Delta}=\left[-\left(\mathcal{M}_{\Delta}^{m} C_{\Delta l}^{\beta r}\right)_{-}, \mathcal{L}_{\Delta}\right]
$$

where $\partial_{l, m, \beta}$ denotes the derivative with respect to an additional new variable $t_{l, m, \beta}^{*}$. The more general actions of the additional symmetry flows of the discrete constrained multi-component $\mathrm{KP}$ are given by

$$
\frac{\partial \mathcal{M}_{\Delta}}{\partial_{m, n, \beta}}=-\left[\left(\mathcal{M}_{\Delta}^{m} C_{\Delta n}^{(\beta, r)}\right)_{-}, \mathcal{M}_{\Delta}\right], \quad \partial_{l, m, \beta} \mathcal{M}_{\Delta}^{n} \mathcal{L}_{\Delta}^{k}=\left[-\left(\mathcal{M}_{\Delta}^{m} C_{\Delta l}^{\beta r}\right)_{-}, \mathcal{M}_{\Delta}^{n} \mathcal{L}_{\Delta}^{k}\right]
$$

Later we can prove the additional flows $\frac{\partial}{\partial t_{m, n, \beta}^{*}}$ commute with the flow $\frac{\partial}{\partial t_{k, \gamma}}$, but do not commute with each other. To this purpose, we need several lemmas and propositions as preparation firstly.

Similarly as differential case, for above local(means only contain non-negative powers on $\Delta$ ) difference operators $B_{\Delta n}^{\beta, r}$, we have the following lemma.

Lemma 5.1. $\left[B_{\Delta n}^{\beta, r}, \phi \hat{\Delta}^{-1} \psi\right]_{-}=B_{\Delta n}^{\beta, r}(\phi) \hat{\Delta}^{-1} \psi-\phi \hat{\Delta}^{-1} B_{\Delta n}^{(\beta, r) *}(\psi)$.

Proof. Firstly we consider a fundamental monomial: $\hat{\Delta}^{n}(n \geq 1)$. Then

$$
\left[\hat{\Delta}^{n}, \phi \hat{\Delta}^{-1} \psi\right]_{-}=\left(\hat{\Delta}^{n}(\phi)\right) \hat{\Delta}^{-1} \psi-\left(\phi \hat{\Delta}^{-1} \psi \hat{\Delta}^{n}\right)_{-} .
$$

Notice that the second term can be rewritten in the following way

$$
\begin{array}{r}
\left(\phi \hat{\Delta}^{-1} \psi \hat{\Delta}^{n}\right)_{-}=\left(\phi\left(\hat{\Lambda}^{-1}(\psi)\right) \hat{\Delta}^{n-1}-\phi \hat{\Delta}^{-1}\left(\hat{\Delta} \Lambda^{-1}(\psi)\right) \hat{\Delta}^{n-1}\right)_{-} \\
=\left(\phi \hat{\Delta}^{-1}\left(-\hat{\Delta} \Lambda^{-1}(\psi)\right) \hat{\Delta}^{n-1}\right)_{-}=\cdots=\phi \hat{\Delta}^{-1}\left(\left(-\hat{\Delta} \Lambda^{-1}\right)^{n}(\psi)\right)=\phi \hat{\Delta}^{-1}\left(\hat{\Delta}^{n}\right)^{*}(\psi),
\end{array}
$$

then the lemma is proved.

We can get some properties of the Lax operator in the following proposition.

By induction, the following important lemma can be derived

Lemma 5.2. The Lax operator $\mathcal{L}_{\Delta}$ of discrete constrained $N$-component KP hierarchy satisfied the relation of

$$
\left(\mathcal{L}_{\Delta}^{k}\right)_{-}=\sum_{j=0}^{k-1} \mathcal{L}_{\Delta}^{k-j-1}(\phi) \hat{\Delta}^{-1}\left(\mathcal{L}_{\Delta}^{*}\right)^{j}(\psi), \quad k \in \mathbb{Z} .
$$

where $\mathcal{L}_{\Delta}(\phi)=\left(\mathcal{L}_{\Delta}\right)_{+}(\phi)+\phi \hat{\Delta}^{-1}(\psi \phi)$. 
Specifically, for example the case when $k=3$,

$$
\left(\mathcal{L}_{\Delta}^{3}\right)_{-}=\left[\mathcal{L}_{\Delta}^{2}(\phi) \hat{\Delta}^{-1} \psi+\mathcal{L}_{\Delta}(\phi) \hat{\Delta}^{-1} \mathcal{L}_{\Delta}^{*}(\psi)+\phi \hat{\Delta}^{-1} \mathcal{L}_{\Delta}^{2 *}(\psi)\right]
$$

According to the definition of the discrete constrained multi-component KP hierarchy, the action of original additional flows of the discrete constrained $N$-component $\mathrm{KP}$ hierarchy is expressed by

$$
\left(\partial_{1, k, \beta} \mathcal{L}_{\Delta}\right)_{-}=\left[\left(\mathcal{M}_{\Delta} C_{\Delta k}^{(\beta, r)}\right)_{+}, \mathcal{L}_{\Delta}\right]_{-}+\left(C_{\Delta k}^{(\beta, r)}\right)_{-} .
$$

Also if $X=M \hat{\Delta}^{-1} N$, then

$$
\left[X, \mathcal{L}_{\Delta}\right]_{-}=\left[M \hat{\Delta}^{-1} \mathcal{L}_{\Delta}^{*}(N)-\mathcal{L}_{\Delta}(M) \hat{\Delta}^{-1} N\right]+\left[X(\phi) \hat{\Delta}^{-1} \psi-\phi \hat{\Delta}^{-1} X^{*}(\psi)\right] .
$$

To keep the consistency with flow equations on wave function $\phi, \psi$, we shall introduce an operator $T_{\Delta k}^{(\alpha, r)}$ to modify the additional symmetry of the discrete constrained multi-component KP hierarchy. We now introduce a pseudo discrete operator $T_{\Delta k}^{(\alpha, r)}$,

$$
\begin{aligned}
T_{\Delta k}^{(\alpha, r)} & =0, \quad k=-1,0,1, \\
T_{\Delta k}^{(\alpha, r)} & =\sum_{j=0}^{k-1}\left[j-\frac{1}{2}(k-1)\right] C_{\Delta k-1-j}^{(\alpha, r)}(\phi) \hat{\Delta}^{-1}\left(C_{\Delta j}^{(\alpha, r) *}\right)(\psi), k \geq 2,
\end{aligned}
$$

and have the following property.

Lemma 5.3. The action of flows $\partial_{t_{l, \beta}}$ of the discrete constrained $N$-component KP hierarchy on the $T_{\Delta k}^{(\alpha, r)}$ is

$$
\partial_{t_{l, \beta}} T_{\Delta k}^{(\alpha, r)}=\left[B_{\Delta l}^{(\beta, r)}, T_{\Delta k}^{(\alpha, r)}\right]_{-} .
$$

Proof. The proof is similar as continuous constrained multi-component KP hierarchy instead of using the Lemma 5.1.

Further, the following expression of $\left[T_{\Delta k-1}^{(\beta, r)}, L\right]_{-}$is also necessary to define the additional flows of the discrete constrained $N$-component KP hierarchy.

Lemma 5.4. The Lax operator $\mathcal{L}_{\Delta}$ of discrete constrained $N$-component KP hierarchy and $T_{\Delta k-1}^{(\beta, r)}$ has the following relation,

$$
\begin{aligned}
{\left[T_{\Delta k-1}^{(\beta, r)}, \mathcal{L}_{\Delta}\right]_{-}=} & -\left(C_{\Delta k}^{(\beta, r)}\right)_{-}+\frac{k}{2}\left[\phi_{\Delta}^{(r)} \hat{\Delta}^{-1}\left(C_{\Delta k-1}^{(\beta, r)}\right)^{*}\left(\psi_{\Delta}^{(r)}\right)+C_{\Delta k-1}^{(\beta, r)}\left(\phi_{\Delta}^{(r)}\right) \hat{\Delta}^{-1} \psi_{\Delta}^{(r)}\right. \\
& \left.+T_{\Delta k-1}^{(\beta, r)}\left(\phi_{\Delta}^{(r)}\right) \hat{\Delta}^{-1} \psi_{\Delta}^{(r)}-\phi_{\Delta}^{(r)} \hat{\Delta}^{-1}\left(T_{\Delta k-1}^{(\beta, r)}\right)^{*}\left(\psi_{\Delta}^{(r)}\right)\right] .
\end{aligned}
$$

Proof. A similar direct calculation as the continuous case can help us deriving eq.(15.18).

We define the additional flows of the discrete constrained $N$-component KP hierarchy as

$$
\partial_{t_{1, k, \beta}^{*}} \mathcal{L}_{\Delta}=\left[-\left(\mathcal{M}_{\Delta} C_{\Delta k}^{(\beta, r)}\right)_{-}+T_{\Delta k-1}^{\beta, r}, \mathcal{L}_{\Delta}\right]
$$


where $T_{\Delta k-1}^{\beta, r}=0$, for $l=0,1,2$, such that the right-hand side of (5.19) is in the form of derivation of Lax equation. Generally, one can also derive

$$
\partial_{t_{1, k, \beta}^{*}}\left(\mathcal{M}_{\Delta} \mathcal{L}_{\Delta}^{l}\right)=\left[-\left(\mathcal{M}_{\Delta} C_{\Delta k}^{(\beta, r)}\right)_{-}+T_{\Delta k-1}^{\beta, r}, \mathcal{M}_{\Delta} \mathcal{L}_{\Delta}^{l}\right] .
$$

Now we calculate the action of the additional flows eq.(5.19) on the eigenfunction $\phi$ and $\psi$ of the discrete constrained $N$-component KP hierarchy.

Theorem 5.5. The acting of additional flows of discrete constrained $N$-component KP hierarchy on the eigenfunction $\phi$ and $\psi$ are

$$
\begin{aligned}
\partial_{t_{1, k, \beta}^{*}} \phi & =\left(\mathcal{M}_{\Delta} C_{\Delta k}^{(\beta, r)}\right)_{+}(\phi)+T_{\Delta k-1}^{(\beta, r)}(\phi)+\frac{k}{2} C_{\Delta k-1}^{(\beta, r)}(\phi), \\
\partial_{t_{1, k, \beta}^{*}} \psi & =-\left(\mathcal{M}_{\Delta} C_{\Delta k}^{(\beta, r)}\right)_{+}^{*}(\psi)-\left(T_{\Delta k-1}^{(\beta, r)}\right)^{*}(\psi)+\frac{k}{2} C_{\Delta k-1}^{(\beta, r) *}(\psi) .
\end{aligned}
$$

Next we shall prove the commutation relation between the additional flows $\partial_{t_{1, k, \beta}^{*}}$ of discrete constrained $N$-component KP hierarchy and the original flows $\partial_{t_{l, \alpha}}$ of it.

Theorem 5.6. The additional flows of $\partial_{t_{1, k, \beta}^{*}}$ are symmetry flows of the discrete constrained $N$-component KP hierarchy, i.e. they commute with all $\partial_{t_{l, \beta}}$ flows.

Proof. According the action of $\partial_{t_{1, k, \beta}^{*}}$ and $\partial_{t_{l, \alpha}}$ on the dressing operator $S_{\Delta}$, then

$$
\begin{aligned}
{\left[\partial_{t_{1, k, \beta}^{*}}, \partial_{t_{l, \alpha}}\right] S_{\Delta} } & =\left[\left(C_{\Delta l}^{(\beta, r)}\right)_{+},-T_{\Delta k-1}^{(\beta, r)}\right]_{-} S_{\Delta}-\left(\partial_{t_{l, \alpha}} T_{\Delta k-1}^{(\beta, r)}\right) S_{\Delta} \\
& =0 .
\end{aligned}
$$

Therefore the theorem holds.

Then using the relation $\partial_{t_{1, l, \beta}^{*}}\left(\mathcal{L}_{\Delta}^{k}(\phi)\right)=\left(\partial_{t_{1, l, \beta}^{*}}\left(\mathcal{L}_{\Delta}^{k}\right)\right)(\phi)+\mathcal{L}_{\Delta}^{k} \partial_{t_{1, l, \beta}^{*}}(\phi)$, we can find the additional flows $\partial_{t_{1, l, \beta}^{*}}$ of discrete constrained $N$-component KP hierarchy have the following relations

$$
\begin{aligned}
\partial_{t_{1, l, \beta}^{*}} \mathcal{L}_{\Delta}^{k}(\phi) & =\left(\mathcal{M}_{\Delta} C_{\Delta l}^{\beta, r}\right)_{+}\left(\mathcal{L}_{\Delta}^{k}(\phi)\right)+\left(k+\frac{l}{2}\right) \mathcal{L}_{\Delta}^{k+l-1}(\phi), \quad l=0,1,2, \\
\partial_{t_{1, l, \beta}^{*}}\left(\mathcal{L}_{\Delta}^{*}\right)^{k}(\psi) & =-\left(\mathcal{M}_{\Delta} C_{\Delta l}^{\beta, r}\right)_{+}^{*}\left(\mathcal{L}_{\Delta}^{*}\right)^{k}(\psi)+\left(k+\frac{l}{2}\right)\left(\mathcal{L}_{\Delta}^{*}\right)^{k+l-1}(\psi), \quad l=0,1,2 .
\end{aligned}
$$

Moreover, the action of $\partial_{t_{1, l, \beta}^{*}}$ on $T_{\Delta k}^{(\alpha, r)}$ is given by the following lemma.

Lemma 5.7. The actions on $T_{\Delta k}^{(\alpha, r)}$ of the additional symmetry flows $\partial_{t_{1, l, \beta}^{*}}$ of the discrete constrained $N$-component $K P$ hierarchy are

$$
\partial_{t_{1, l, \beta}^{*}} T_{\Delta k}^{(\alpha, r)}=\left[\left(\mathcal{M}_{\Delta} C_{\Delta l}^{\beta, r}\right)_{+}+T_{\Delta l-1}^{(\beta, r)}, T_{\Delta k}^{(\alpha, r)}\right]_{-}+(k-l+1) T_{\Delta k+l-1}^{(\alpha, r)} \delta_{\alpha, \beta} .
$$

Proof. Using the same technique as continuous case, a straightforward calculation implies eq. (5.23).

Now it is time to identity the algebraic structure of the additional symmetry flows of the discrete constrained $N$-component KP hierarchy. 
Theorem 5.8. The additional flows $\partial_{t_{1, k, \beta}^{*}}$ of the discrete constrained $N$-component KP hierarchy form the positive half of Virasoro algebra, i.e., for $l=0,1,2 ; k \geq 0$,

$$
\left[\partial_{t_{1, l, \alpha}^{*}}, \partial_{t_{1, k, \beta}^{*}}\right]=(k-l) \delta_{\alpha, \beta} \partial_{t_{1, k+l-1, \alpha}^{*}} .
$$

Proof. Using $\left[\left(\mathcal{M}_{\Delta} C_{\Delta l}^{\alpha, r}\right)_{-}, T_{\Delta k-1}^{(\beta, r)}\right]_{-}=\left[\left(\mathcal{M}_{\Delta} C_{\Delta l}^{\alpha, r}\right)_{-}, T_{\Delta k-1}^{(\beta, r)}\right]$ and the Jacobi identity, one can derive the following computation

$$
\begin{aligned}
& {\left[\partial_{t_{1, l, \alpha}^{*}}, \partial_{t_{1, k, \beta}^{*}}\right] \mathcal{L}_{\Delta} } \\
= & \partial_{t_{1, l, \alpha}^{*}}\left(\left[-\left(\mathcal{M}_{\Delta} C_{\Delta k}^{\beta, r}\right)_{-}, \mathcal{L}_{\Delta}\right]+\left[T_{\Delta k-1}^{(\beta, r)}, \mathcal{L}_{\Delta}\right]\right)-\partial_{t_{1, k, \beta}^{*}}\left(\left[-\left(\mathcal{M}_{\Delta} C_{\Delta l}^{\alpha, r}\right)_{-}, \mathcal{L}_{\Delta}\right]\right) \\
= & \partial_{t_{1, l, \alpha}^{*}}\left[-\left(\mathcal{M}_{\Delta} C_{\Delta k}^{(\beta, r)}\right)_{-}, \mathcal{L}_{\Delta}\right]+\left[\partial_{t_{1, l, \alpha}^{*}} T_{\Delta k-1}^{(\beta, r)}, \mathcal{L}_{\Delta}\right]+\left[T_{\Delta k-1}^{(\beta, r)}, \partial_{t_{1, l, \alpha}^{*}} \mathcal{L}_{\Delta}\right]+\left[\partial_{t_{1, k, \beta}^{*}}\left(\mathcal{M}_{\Delta} C_{\Delta l}^{\alpha, r}\right)_{-}, \mathcal{L}_{\Delta}\right] \\
& +\left[\left(\mathcal{M}_{\Delta} C_{\Delta l}^{\alpha, r}\right)_{-}, \partial_{t_{1, k, \beta}^{*}} \mathcal{L}_{\Delta}\right] \\
= & {\left[-\left(\partial_{t_{1, l, \alpha}^{*}}\left(\mathcal{M}_{\Delta} C_{\Delta k}^{(\beta, r)}\right)\right)_{-}, \mathcal{L}_{\Delta}\right]+\left[-\left(\mathcal{M}_{\Delta} C_{\Delta k}^{\beta, r}\right)_{-},\left(\partial_{t_{1, l, \alpha}^{*}} \mathcal{L}_{\Delta}\right)\right]+\left[\left[\left(\mathcal{M}_{\Delta} C_{\Delta l}^{\alpha, r} R_{\Delta \beta}\right)_{+}, T_{\Delta k-1}^{(\beta, r)}\right]_{-}\right.} \\
& \left.+(k-l) T_{k+l-2}^{(\beta, r)} \delta_{\alpha, \beta}, \mathcal{L}_{\Delta}\right]+\left[T_{\Delta k-1}^{(\beta, r)},\left[-\left(\mathcal{M}_{\Delta} C_{\Delta l}^{\alpha, r}\right)_{-}, \mathcal{L}_{\Delta}\right]\right]+\left[\left[-\left(\mathcal{M}_{\Delta} C_{\Delta k}^{(\beta, r)}\right)_{-}\right.\right. \\
& \left.\left.+T_{\Delta k-1}^{(\beta, r)}, \mathcal{M}_{\Delta} C_{\Delta l}^{\alpha, r}\right]_{-}, \mathcal{L}_{\Delta}\right]+\left[\left(\mathcal{M}_{\Delta} C_{\Delta l}^{\alpha, r}\right)_{-},\left[-\left(\mathcal{M}_{\Delta} C_{\Delta k}^{(\beta, r)}\right)_{-}+T_{\Delta k-1}^{(\beta, r)}, \mathcal{L}_{\Delta}\right]\right] \\
= & (k-l)\left[-\left(\mathcal{M}_{\Delta} \mathcal{L}_{\Delta}^{k+l-1}\right)_{-}, \mathcal{L}_{\Delta}\right]+(k-l)\left[\delta_{\alpha, \beta} T_{k+l-2}, \mathcal{L}_{\Delta}\right] \\
& -\left[\left[\left(\mathcal{M}_{\Delta} C_{\Delta l}^{\alpha, r}\right)_{-}, T_{\Delta k-1}^{(\beta, r)}\right], \mathcal{L}_{\Delta}\right]+\left[T_{\Delta k-1}^{(\beta, r)},\left[-\left(\mathcal{M}_{\Delta} C_{\Delta l}^{\alpha, r}\right)_{-}, \mathcal{L}_{\Delta}\right]\right]+\left[\left(\mathcal{M}_{\Delta} C_{\Delta l}^{\alpha, r}\right)_{-},\left[T_{\Delta k-1}^{(\beta, r)}, \mathcal{L}_{\Delta}\right]\right] \\
= & (k-l) \delta_{\alpha, \beta} \partial_{t_{1, k+l-1, \alpha}^{*}} \mathcal{L}_{\Delta} .
\end{aligned}
$$

This shows that the discretization from constrained KP hierarchy to discrete constrained $N$-component KP hierarchy keeps the important virasoro algebraic structure.

\section{Conclusions and Discussions}

In this paper, the additional symmetry flows in eq.(3.17) for the constrained $N$-component KP hierarchy have been constructed. In this process, with the help of differential operator

$T_{k}^{(\alpha, r)}$, the additional flows (3.17) on eigenfunctions and adjoint eigenfunctions of constrained $N$-component KP hierarchy were obtained in Theorem 3.6. In Theorem 3.9, these flows have been shown to provide a hidden algebraic structure, i.e., the Virasoro algebra. Also we construct the additional symmetries and prove a lot of similar results of the discrete constrained multicomponent KP hierarchy and give the Virasoro flow equations on eigenfunctions and adjoint eigenfunctions. This tells us that the discretization from constrained KP hierarchy to discrete constrained $N$-component KP hierarchy retain the important virasoro algebraic structure.

Acknowledgments Chuanzhong Li is supported by the National Natural Science Foundation of China under Grant No. 11201251, 11571192, the Zhejiang Provincial Natural Science Foundation under Grant No. LY15A010004, LY12A01007, the Natural Science Foundation of Ningbo under Grant No. 2015A610157. Jingsong He is supported by the National Natural Science Foundation of China under Grant No. 11271210, K. C. Wong Magna Fund in Ningbo University. 


\section{REFERENCES}

[1] L. Dickey, Additional symmetries of KP, Grassmannian, and the string equation, Modern Physics Letters A, 8(1993), 1259-1271.

[2] B. G. Konopelchenko, J. Sidorenko and W. Strampp, $(1+1)$-dimensional integrable systems as symmetry constraints of (2+1)-dimensional systems, Phys. Lett. A 157(1991), 17-21.

[3] Y. Cheng and Y. S. Li, The constraint of the Kadomtsev-Petviashvili equation and its special solutions, Phys. Lett. A 157 (1991), 22-26.

[4] Y. Cheng, Constraints of the Kadomtsev-Petviashvili hierarchy, J. Math. Phys. 33(1992), 3774-3782.

[5] H. Aratyn, E. Nissimov and S. Pacheva, Virasoro symmetry of constrained KP Hierarchies, Phys. Lett. A 228(1997), 164-175.

[6] C. Z. Li, J. S. He, Y. C. Su, Block type symmetry of bigraded Toda hierarchy, J. Math. Phys. 53(2012), 013517.

[7] C. Z. Li, J. S. He, Block algebra in two-component BKP and D type Drinfeld-Sokolov hierarchies, J. Math. Phys. 54(2013), 113501.

[8] C. Z. Li, J. P. Cheng etal, Ghost symmetry of the discrete KP hierarchy, Monatshefte Fuer Mathematik, 180(2016), 815-832.

[9] M. H. Li, C. Z. Li etal, Virasoro type algebraic structure hidden in the constrained discrete KadomtsevPetviashvili hierarchy, J. Math. Phys. 54(2013), 043512.

[10] L. Dickey, Additional symmetries of KP, Grassmannian, and the string equation II, Modern Physics Letters A, 8(1993), 1357-1377.

[11] V. G. Kac and J. W. van de Leur, The n-component KP hierarchy and the representation theory Important Developments in Soliton Theory (Springer Series in Nonlinear Dynamics)(1993), 302.

[12] A. Maffei, The Multicomponent KP and Fay Trisecant Formula, International Mathematics Research Notices 16(1996), 769-791.

[13] Y. Zhang, On a reduction of the multi-component KP hierarchy, J. Phys. A: Math. Gen. 32(1999), 6461-6476.

[14] M. Adler, P. van Moerbeke, P. Vanhaecke, Moment Matrices and Multi-Component KP, with Applications to Random Matrix Theory Commun. Math. Phys. 286(2009), 1-38.

[15] C. Álvarez Fernández, U. Fidalgo Prieto, and M. Mañas, Multiple orthogonal polynomials of mixed type: Gauss-Borel factorization and the multi-component 2D Toda hierarchy, Advances in Mathematics, 227(2011), 1451-1525.

[16] C. Z. Li, J. S. He, The extended multi-component Toda hierarchy, Math. Phys. Analyis and Geometry. 17(2014), 377-407.

[17] C. Z. Li, J. S. He, The extended $Z_{N}$-Toda hierarchy, Theoretical and Mathematical Physics, 185(2015), 1614-1635.

[18] B. A. Kupershimidt, Discrete Lax equations and differential-difference calculus, Asterisque, 123(1985), $1-212$.

[19] S. K. Vel and K. M. Tamizhmani, Lax Pairs, Symmetries and Conservation Laws of a DifferentialDifference Equation-Sato's Approach, Chaos, Solitons and Fractals, 8(1997) 917-931.

[20] L. Haine and P. Iliev, Commutative rings of difference operators and an adelic flag manifold, Int. Math. Res. Not. 6(2000), 281-323.

[21] S. W. Liu, Y. Cheng, Sato Backlund transformation, additional symmtries and ASvM formular for the discrete KP hierarchy, J. Phys. A: Math. Theor., 43(2010),135202.

[22] A. Yu. Orlov, E. I. Schulman, Additional symmetries of integrable equations and conformal algebra reprensentaion, Lett. Math. Phys. 12(1986), 171-179.

[23] Y. Cheng and Y. J. Zhang, Solutions for the vector k-constrained KP hierarchy, J. Math. Phys. 35(1994), 5869 . 
[24] L. Fehér and I. Marshallm, Extensions of the matrix Gelfand-Dickey hierarchy from generalized Drinfeld-Sokolov reduction Commun. Math. Phys. 183( 1997), 423.

[25] M. F. de Groot, T. J. Hollowood and J. L. Miramontes, Generalized Drinfeld-Sokolov hierarchies Commun. Math. Phys. 145(1992), 57. 